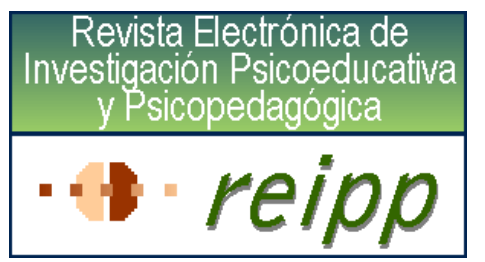

REVISTA ELECTRÓNICA DE

INVESTIGACIÓN PSICOEDUCATIVA Y

PSICOPEDAGÓGICA

No 1 (2) 2003. ISSN: 1696-2095

\title{
Solución de Problemas: Estudio evaluativo de tres enfoques pedagógicos en las escuelas mexicanas
}

\author{
María Teresa Esquivias Serrano* \\ Arturo González Cantú** \\ Irene Muria Vila*** \\ * Instituto Tecnológico y de Estudios Superiores de Monterrey \\ ** Universidad Autónoma de Nuevo León \\ *** Universidad Nacional Autónoma de México \\ México
}

mtesquiv58@hotmail.com

argonzal@hotmal.com

imuria@servidor.unam.mx 


\section{RESUMEN}

Introducción. Una de las metas más importantes del sistema educativo mexicano, es que los alumnos adquieran desde su formación primaria, habilidades de pensamiento complejo, como son: la "solución de problemas" y la "creatividad" entre otras. El presente trabajo describe de qué manera la "solución de problemas" se manifiesta en tres los diferentes enfoques pedagógicos en México.

Método. El diseño utilizado es de tipo no experimental porque que se realiza sin manipular deliberadamente las variables observando así una situación existente, y transeccional o transversal descriptivo, porque la medición se lleva a cabo en una sola ocasión, obteniendo la incidencia de valores en que se manifiesta la variable estudiada. Se sustenta además con un diseño de tipo mixto 6 X 1 .

Resultados. Los resultados obtenidos muestran cómo los niños de la escuela Freinet obtienen las puntuaciones más altas en ambos grupos y las puntuaciones más bajas corresponden a la escuela tradicional oficial. Existen diferencias estadísticamente significativas entre los diferentes enfoques pedagógicos, en lo que respecta a la solución de problemas en su proceso educativo básico.

Discusión. Se requiere un nuevo modelo de enseñanza, que contemple todas las potencialidades del ser humano en los programas, ajustando los recursos humanos y materiales necesarios responsables del proceso educativo. Resulta indispensable incluir contenidos educativos donde se ejercite, enseñe y desarrolle las habilidades para "solucionar problemas".

Palabras clave: Solución de problemas, Enfoques pedagógicos, Educación Tradicional, Montessori y Freinet, Educación Básica. 


\section{Introducción}

En la reforma educativa realizada en México Programa para la Modernización Educativa (SEP, 1989-1994), se establece como uno de los objetivos fundamentales de la Educación Básica, “aprender a resolver problemas". Sin embargo, no existe en este momento, un proceso de evaluación formal y sistemático que nos permita saber si esta habilidad del pensamiento de orden superior, se logra en los niveles deseados. Es importante mencionar entonces, que en la investigación que presentamos, se evalúa uno de los procesos cognitivos más complejos como es: la "solución de problemas", en tres enfoques pedagógicos que prevalecen en nuestra sociedad. Consideramos que este trabajo de investigación es relevante tanto en el aspecto educativo como social en nuestro país, debido a que la evaluación de la solución de problemas en sus educandos en tres aproximaciones diferentes, permitirá entender cuál de ellos favorece este tipo de procesos, además de establecer de alguna manera una forma de evaluación de este proceso cognitivo.

La "solución de problemas" es la forma más elevada del aprendizaje (Klausmeier y Goodwin, 1993), debido a que a partir de este proceso el individuo define nuevas ideas. Asimismo, es sabido que frente a un problema se requiere por un lado del conocimiento de reglas, y por el otro de capacidad para utilizarlas, logrando así transferencias de lo aprendido. Por lo que ser capaz de resolver problemas habilita a las personas para adaptarse al medio ambiente y para modificarlo de manera parcial.

Hablar de solución de problemas, es hablar de "habilidades de pensamiento", siendo estas habilidades de las expresiones más elevadas y complejas en el ser humano, lo cual implica además de los procesos mentales superiores, procesos más sencillos tales como la memoria, la atención, representación, comprensión,...etc. En el pensamiento se generan actividades mentales como la articulación de símbolos y conceptos, los cuales nos dirigen a la creación de formas nuevas que culminan en la "solución de problemas". Al referirnos a la solución de problemas, a lo largo de este trabajo, lo haremos como (S.P.).

Para De Vega (1986, p. 494), la definición de problema se encierra en: “...aquellas tareas que exigen de procesos de razonamiento relativamente complejos, y no una mera actividad asociativa y rutinaria". De este modo el proceso que se lleva a cabo en la S. P. puede ser manejado como una actividad dirigida hacia una meta, la cual en ocasiones es rutinaria, 
utilizando lo que ya existe (reproducción), o en otras creativa generando nuevos procedimientos (producción).

Para Pozo (Pozo, Pérez, Domínguez, Gómez y Postigo, 1994; p. 9), “La solución de problemas se basa en el planteamiento de situaciones abiertas y sugerentes que exijan de los alumnos una actitud activa y un esfuerzo por buscar sus propias respuestas, su propio conocimiento". La S. P. es en sí, un proceso en el cual se interrelacionan elementos externos, (problemas a solucionar), con los internos ya existentes en el sujeto, (memoria, reglas simples, reglas complejas,...etc.), denominadas estrategias cognoscitivas (habilidades intelectuales), para obtener de esta interacción la solución adecuada al problema en cuestión, y la modificación de la capacidad intelectual de una persona, dado que al solucionar el problema se ha logrado obtener una "regla de orden superior", la cual se agrega y permanece en el repertorio del individuo. Enseñar a resolver problemas fomenta en los alumnos la capacidad de aprender a aprender. Los alumnos necesitan adquirir habilidades y estrategias que les permitan aprender por sí mismos nuevos conocimientos. (Pozo et al., 1994).

Es entonces cuando se puede hablar de "aprendizaje" al hablar de "solución de problemas" y de las capacidades involucradas en esta actividad, las cuales son: las habilidades intelectuales, la información verbal organizada y las estrategias cognoscitivas que logre articular una persona en este proceso.

Por lo tanto, la S. P., queda definida en términos de la presente investigación de la siguiente forma: "La solución de problemas es un proceso mental elevado en el que intervienen: actitudes, conocimientos previos, reglas heurísticas o algorítmicas, para aportar la mejor de las respuestas a un problema, donde no la había” (Esquivias, 1997).

Acuña y Batllori (1988) haciendo referencia a varios autores tales como Wylie, Samson, De Bono, Maier, Dunker, Polya , Krulik y Rudnik, y Anderson y Barry, explican que todos ellos coinciden en que la solución de problemas es un proceso que requiere de cuatro pasos: 1) identificación del problema, 2) formulación del mismo, 3) aplicación de datos a cada solución y 4) selección de la mejor alternativa.

Por otro lado, algunas investigaciones realizadas dentro del campo educativo, muestran que el estudiante no sigue una secuencia consistente en un proceso de solución de 
problemas, debido a que carece del hábito de aplicación de la lógica y el razonamiento. (Sánchez, 1996).

La S.P. debería de ser entendida como parte fundamental de cualquier proceso educativo, de forma que al final de cualquier trayectoria profesional, técnica, etc. somos todos solucionadores de problemas, en una forma o en otra, en mayor o en menor grado y el contar con elementos y/o habilidades de pensamiento lógico y de razonamiento, nos permitirá un adecuado desempeño en el ejercicio de nuestra profesión o actividad que realicemos.

Piaget y García (1983; citado en Pozo,1999), muestran que los nuevos conceptos surgen generalmente por integración de algunos más simples, resaltando que de la reorganización de ideas se adquieren nuevos significados, por lo que el papel que se le confiere al educando es determinante de la construcción de su propio conocimiento. Para Ausubel (Ausubel et al, 1998, p. 463), "la capacidad para resolver problemas es la meta primordial de la educación", conocida esta proposición como la quinta de la tesis del aprendizaje por descubrimiento, soportando de esta manera la importancia del proceso en los estudiantes. Las aportaciones de Vygotsky al respecto van encaminadas a resaltar la cooperación en el trabajo grupal, principio que se deriva de los postulados teóricos del paradigma sociocultural de este autor. Por lo tanto las posturas psicoeducativas cognitiva y constructivista, consideran dentro de sus propuestas, la "solución de problemas" como una capacidad determinante en el proceso formativo de cualquier individuo.

\section{Enfoques pedagógicos}

En cuanto a los enfoques pedagógicos revisados, encontramos que los distinguen características específicas a cada uno de ellos. Donde difieren desde su filosofía, su metodología, sus materiales a utilizar, entre otros aspectos. Los abordaremos brevemente.

\section{1) Tradicional:}

Es el que se imparte en la mayoría de escuelas en nuestro país, se encuentra centrado en la transmisión del conocimiento de tipo declarativo, favoreciendo un aprendizaje por recepción-repetitivo, en donde el papel principal lo desempeña el maestro, como única autoridad ante el grupo. El profesor es orador, transmisor del conocimiento, y el alumno es auditorio, es receptor pasivo de dicho conocimiento. La democracia difícilmente se propicia en estos espacios, el profesor es el que toma las decisiones respecto al grupo en general. 
2) Montessori:

Esta escuela se inscribe en lo que se consideraba en su tiempo como Pedagogía Científica, cuya concepción de la educación se inspira en la "naturaleza y en las leyes del desarrollo infantil, al margen de los hábitos tradicionales, incluso de la propia tradición, y de toda consideración metafísica" (Monés i Pujol-Busquets 2000 p.24).

Los principios educativos de este método son los siguientes:

"Potenciar la actividad motriz y la percepción sensorial como fuentes principales de aprendizaje y para el desarrollo del niño.

$>$ Dar importancia a la educación artística como medio de expresión del mundo interior y de comunicación con la realidad exterior.

$>$ Crear un método de lecto-escritura basado en la lógica y que evite el aprendizaje mecánico.

> Familiarizar al niño con las matemáticas, hacer que las sienta próximas, accesibles, formando parte de su mundo.

$>$ Ayudar al niño a experimentar, observar y clasificar lo que percibe de su entorno físico y social." (Vallet, 2000; p.33).

Aquí el alumno es el protagonista del proceso enseñanza-aprendizaje, trabajando a su propio ritmo en un ambiente libre con materiales especializados y elaborados específicamente para ello, donde el mobiliario corresponde a la etapa de su desarrollo físico y mental. El papel del profesor es principalmente un guía y asesor de los niños.

\section{3) Freinet:}

Una de las características centrales de este tipo de pedagogía es precisamente el "socialismo". Aquí Freinet aporta un concepto con importantes implicaciones, denominando a la clase "célula social" y convirtiéndola en una institución democrática y cooperativa en donde el tanteo experimental permite al alumno confrontarse y abordar dificultades, las cuales conducen al intercambio y a la cooperación (Freinet, 1985). "El concepto de funcionalismo adquiere en Freinet una dimensión extraordinaria: todas sus técnicas están al servicio de la capacidad de experimentación y de expresión de los niños, para la solución de sus necesidades inmediatas" (Vilaplana, 2000; p.72).

En este enfoque, los alumnos son los protagonistas en el salón de clase, aquí ellos junto con el profesor y de manera democrática, toman las decisiones sobre lo que van 
aprender y en algunas ocasiones sobre la forma de hacerlo. La socialización es un factor fundamental en el aula Freinet, la conciencia social que se genera en este ambiente proporciona los elementos para una mejor convivencia con los demás.

\section{Objetivos}

El propósito de esta investigación se sintetiza en los siguientes objetivos:

1) proporcionar datos reales que nos aporten información sobre la manera en que se manifiesta el proceso de "solución de problemas" en las diferentes aproximaciones pedagógicas estudiadas

2) determinar qué enfoque facilita más este proceso, para tratar de entender que elementos o características representativas de cada una de las aproximaciones, contribuye a desarrollar esta capacidad en los educandos

3) resaltar la importancia de incluir contenidos, estrategias y metodologías en general encaminadas al desarrollo de estas capacidades en los estudiantes, tratando de crear conciencia y sembrando inquietud en cuanto a su atención, alentando de esta manera, una reconceptualización de la educación en nuestro país

4) establecer relaciones entre la teoría y la práctica educativa, a partir de las propuestas en los documentos oficiales que sustentan la educación en nuestro país.

\section{Metodología}

\section{Muestra}

La muestra es no probabilística de tipo intencionado (conveniencia), debido a que se seleccionaron los elementos que se consideraron representativos para la finalidad de este estudio (Salkind, 1998). Asimismo, está conformada por 259 niños y niñas inscritos en el tercer (evaluación formativa) y sexto grados (evaluación sumativa) de la educación primaria. Y se llevó a cabo de la siguiente manera:

1) Por ser las más representativas de cada uno de los enfoques estudiados (prestigio).

2) Por nivel socioeconómico (controlado por la ubicación y por corresponder a un rango de colegiatura de entre 10 o 12 salarios mínimos).

3) Los grupos fueron asignados por los directivos en la mayoría de las escuelas, mencionando que eran de los mejores grupos (informe verbal).

4) Fueron seleccionadas 5 escuelas, trabajando con dos grupos por escuela lo que nos da un total de 10 grupos. Cabe mencionar aquí, que la selección de una escuela en la zona 
norte (diferente a todas las demás escuelas localizadas en la zona sur), fue precisamente con la finalidad de comparar los resultados. De esta forma la población estudiada quedó conformada como se indica en la Tabla 1.

Tabla 1

Muestra de la Población estudiada

\begin{tabular}{lrrrrr}
\hline Escuela & Niñas 3 $^{\circ}$ & Niñas 6 $^{\circ}$ & Niños 3 $^{\circ}$ & Niños 6 $^{\circ}$ & Total \\
\hline Freinet & 18 & 13 & 11 & 20 & 62 \\
Montessori & 13 & 13 & 5 & 4 & 35 \\
Trad. Part. & 4 & 12 & 16 & 19 & 51 \\
Trad. Oficial N & 12 & 11 & 8 & 10 & 41 \\
Trad. Oficial S & 19 & 16 & 22 & 13 & 70 \\
Total & $\mathbf{6 6}$ & $\mathbf{6 5}$ & $\mathbf{6 2}$ & $\mathbf{6 6}$ & $\mathbf{2 5 9}$ \\
& & & & & \\
\hline
\end{tabular}

\section{Escenario}

El escenario consistió en los salones de clase de las cinco escuelas mencionadas, las características de cada una de ellas se muestran en la Tabla 2 .

Tabla 2

Características de las Escuelas Estudiadas

\begin{tabular}{ll}
\hline Escuela & Enfoque \\
\hline $\mathrm{N}^{\circ} 1$ & Freinet \\
$\mathrm{N}^{\circ} 2$ & Montessori \\
$\mathrm{N}^{\circ} 3$ & Tradicional Particular \\
$\mathrm{N}^{\circ} 4$ & Tradicional Oficial (Sur) \\
$\mathrm{N}^{\circ} 5$ & Tradicional Oficial (Norte) \\
\hline
\end{tabular}

\section{Variables}

Las variables estudiadas quedaron definidas de la siguiente manera:

Variable independiente $=$ enfoque pedagógico: Tradicional, Montessori y Freinet.

Variable dependiente $=$ Solución de Problemas

\section{Instrumentos}

Los materiales utilizados fueron dos instrumentos elaborados por Esquivias (1997), denominados Instrumentos de "Solución de Problemas $3^{\circ}$ y $6^{\circ}$ grados". Previamente a su 
aplicación definitiva, se realizaron dos estudios piloto y se realizaron las modificaciones y ajustes correspondientes. En cuanto a la fiabilidad del instrumento, se realizó la prueba estadística Alfa de Cronbach, la cual permite determinar la consistencia interna de los reactivos. En esta prueba se obtuvo un coeficiente de fiabilidad Alpha de .6831, y un Alpha estandarizado de .6905 , lo cual indica una consistencia significativa estadísticamente de los reactivos que conforman el instrumento utilizado para la evaluación de esta variable. Los instrumentos están formados por cinco cuartillas que contienen 15 reactivos de opción múltiple cada uno, con dos formatos distintos correspondientes al grado de dificultad para cada nivel educativo estudiado $3^{\circ}$ y $6^{\circ}$. A continuación se presenta un reactivo de cada tipo de examen para ejemplificarlo.

\section{Ejemplo del reactivo No. 14 del Examen $3^{\text {er }}$. Grado}

14. - Imagínate que te encuentras frente a una escalera que tiene 10 escalones y se te pide que subas 4 escalones, después que bajes 1 , después que subas 4 ¿en qué número de escalón te quedaste?
A) Escalón no. 7
B) Escalón no.10
C) Escalón no. 8
D) Escalón no. 5
E) Escalón no. 6

\section{Ejemplo del reactivo No. 14 del Examen $6^{\circ}$. Grado}

14. - Indica cual grupo proporciona los valores correctos de cada una de las letras de la siguiente operación:

operación TERE donde: $\mathrm{T}=3$

$$
\frac{\text { TONO }}{\text { LULU }}
$$

A) $\mathrm{T}=3, \mathrm{~L}=6, \tilde{\mathrm{N}}=8, \mathrm{R}=4, \mathrm{E}=7, \mathrm{U}=5, \mathrm{O}=2$

B) $\quad \mathrm{T}=3, \mathrm{O}=0, \mathrm{E}=1, \mathrm{U}=1, \tilde{\mathrm{N}}=5, \mathrm{~L}=4, \mathrm{R}=6$

C) $\mathrm{T}=3, \mathrm{R}=4, \mathrm{~L}=8, \tilde{\mathrm{N}}=2, \mathrm{O}=1, \mathrm{U}=5, \mathrm{E}=7$

D) $\quad \mathrm{T}=3, \mathrm{U}=8, \mathrm{O}=1, \tilde{\mathrm{N}}=2, \mathrm{E}=7, \mathrm{~L}=6, \mathrm{R}=4$

E) $\mathrm{T}=3, \tilde{\mathrm{N}}=5, \mathrm{E}=7, \mathrm{O}=1, \mathrm{R}=6, \mathrm{~L}=0, \mathrm{U}=8$

\section{Diseño}

El diseño utilizado es de tipo no experimental debido a que se realiza sin manipular deliberadamente las variables, observando así una situación existente y transeccional o transversal descriptivo, porque la medición se lleva a cabo en una sola ocasión, obteniendo la incidencia de valores en que se manifiesta la variable a ser estudiada. (Hernández et al, 1991). 
Se sustenta además con un diseño de tipo mixto 6 X 1 (Cook y Campbell, 1979) con el fin de observar la relación existente entre las variables involucradas en la investigación y por lo tanto los efectos en la variable dependiente S. P.

\section{Procedimiento}

Etapas:

1) Esta investigación se llevó a cabo con la participación de alumnos de los tres enfoques pedagógicos mencionados. Siendo cinco escuelas en total las que formaron parte de esta investigación.

2) En el momento de la aplicación de los instrumentos se solicitó al profesor, que permaneciera en el aula, sin participar ni comunicarse con sus alumnos, con la finalidad de no alterar al grupo y darle confianza de tener la presencia de su maestro en el grupo.

3) En todos los casos fue el mismo aplicador de instrumentos. La aplicación se realizó una vez por grupo, en un intervalo de la primera escuela a la última, de tres semanas.

4) Se aplicaron dos instrumentos diferentes correspondientes al nivel académico aplicado $3^{\circ}$ y $6^{\circ}$ grados por escuela.

5) Se calificaron cada uno de los 259 instrumentos aplicados, de forma objetiva.

6) Se les dio un orden categórico, por escuelas, grados y puntajes obtenidos.

7) Se realizó la codificación de los datos.

8) Se obtuvieron promedios brutos. Se dio orden decreciente de acuerdo a los puntajes obtenidos.

9) Se obtuvo una calificación final del instrumento de S.P. la cual fue obtenida multiplicando cada uno de los promedios brutos por $2 / 3$ (.66666) para hacer equivalente el 15 (número de respuestas correctas), a la escala de 1 a 10.

10) Las comparaciones de promedios obtenidos corresponden a la suma total de ambos puntajes de los grupos evaluados por escuela $3^{\circ}$ y $6^{\circ}$ grados por enfoque, obteniendo un puntaje final.

11) Se efectuó el análisis estadístico correspondiente, que incluye un análisis estadístico de tipo descriptivo, con frecuencias, medias y desviación estándar.

12) También se realizó un análisis estadístico de tipo comparativo el cual incluye: análisis de varianza y correlación entre variables, así como sus respectivos histogramas.

13) Se realizó la prueba estadística Alfa de Cronbach, para determinar la consistencia interna de los reactivos del instrumento. 
14) Para el análisis estadístico de los datos se utilizó el paquete estadístico SPSS (Statistical package of Social Sciences) versiones: 7.0, 8.01 y 10.

15) Se obtuvieron los resultados y se generaron la discusión y las conclusiones correspondientes.

\section{Resultados}

En la tabla 3 y en la gráfica 1, podemos observar claramente cómo los niños de la escuela Freinet obtienen las puntuaciones más altas tanto en el grupo de tercero como de sexto grados, y las más bajas corresponden a la escuela tradicional oficial.

Tabla 3

\section{Resumen del Análisis Estadístico}

\begin{tabular}{lllll}
\hline ESCUELA & GRUPO & $\mathbf{N}^{\mathbf{0}}$ DE ALUMNOS & MEDIA & DES. EST. \\
\hline Freinet & $3^{\circ}$. & 29 & 7.838 & 1.470 \\
& $6^{\circ}$. & 33 & 8.882 & 1.101 \\
Montessori & $3^{\circ}$. & 18 & 5.772 & 2.134 \\
& $6^{\circ}$. & 17 & 8.659 & .870 \\
Trad. Part. & $3^{\circ}$. & 20 & 5.930 & 1.664 \\
& $6^{\circ}$. & 31 & 7.335 & 1.289 \\
Trad. Norte & $3^{\circ}$. & 20 & 4.210 & 1.686 \\
& $6^{\circ}$. & 21 & 6.729 & 1.301 \\
Trad. Sur & $3^{\circ}$. & 41 & 5.788 & 1.901 \\
& $6^{\circ}$. & 29 & 6.455 & 1.697 \\
\hline
\end{tabular}

\section{Gráfica 1}

Solución de problemas ambos grupos $3^{\circ}$ y $6^{\circ}$. Por enfoque

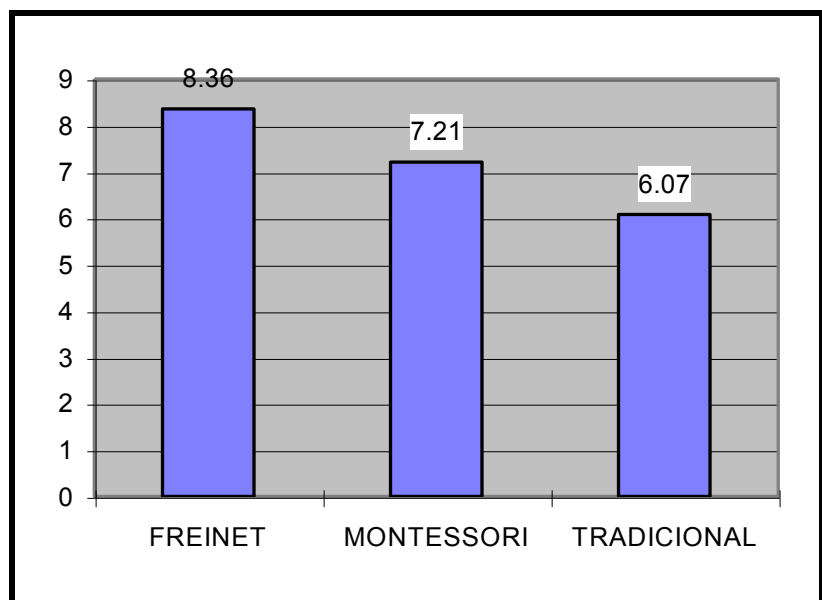


En la tabla 4 se muestran las diferencias estadísticamente significativas entre los grupos estudiados, con un nivel de significación de .05 ó más, donde $\left(^{*}\right)$ representa los pares significativamente diferentes en cuanto a sus resultados.

Tabla 4

Análisis de Varianza Solución de problemas

\begin{tabular}{lllllll}
\hline ESCUELA & MEDIA & $\mathbf{5}$ & $\mathbf{4}$ & $\mathbf{3}$ & $\mathbf{2}$ & $\mathbf{1}$ \\
\hline Freinet & 15.0581 & $*$ & $*$ & $*$ & $*$ & \\
Montessori & 10.7804 & $*$ & & & & \\
Trad. Part. & 10.7600 & $*$ & & & & \\
Trad. Of. Norte & 10.4683 & $*$ & & & & \\
Trad. Of. Sur & 8.0514 & & & & & \\
\hline
\end{tabular}

Los grados de libertad expresados en total (entre grupos y dentro de los grupos), Tabla 5, son de 258. Los resultados obtenidos en la escuela Freinet son diferentes con un nivel de significancia de .05 o más con respecto a todos los demás grupos, tanto de la escuela Montessori como la escuela correspondiente al enfoque Tradicional.

\section{Tabla 5}

Datos del Análisis de Varianza

\begin{tabular}{lrlrc} 
& Sum of & Mean & F & F \\
Source & DF & Squares & Squares & Ratio Prob \\
\hline Between Groups & 4 & 1645.6052 & 411.4013 & 64.1718 .0000 \\
\hline Within Groups & 254 & 1628.3790 & 6.4109 & \\
\hline Total & $\mathbf{2 5 8}$ & $\mathbf{3 2 7 3 . 9 8 4 2}$ & &
\end{tabular}

Existen diferencias estadísticamente significativas entre los enfoques pedagógicos analizados, en lo que respecta a la solución de problemas en su proceso educativo formativo (Tabla No. 4 Análisis de varianza de la variable S. P.).

La escuela que obtuvo las puntaciones más altas en el instrumento de razonamiento S.P. en ambos grupos $3^{\circ}$ y $6^{\circ}$, es la escuela que se rige bajo las técnicas "Freinet", con una media de 8.36 .

La escuela que se ubica después, es la escuela de tipo "Montessori", con una media de 7.21 .

La escuela "tradicional particular", en tercer lugar en cuanto a puntuaciones obtenidas en la muestra, con una media de 6.63. 
Existen diferencias significativas estadísticamente hablando de la escuela Freinet, comparada con las otras y diferencias entre las escuelas Montessori y Tradicional particular, lo cual sugiere realizar más estudios y serios análisis futuros entre estos enfoques.

Las escuelas "tradicionales" oficiales (las dos restantes, tanto norte como sur), son las que muestran las menores puntaciones en razonamiento, siendo de 5.47 y 6.12 sus medias resultantes.

Al promediar las medias de S.P. de las tres últimas escuelas obtenemos la puntación del Enfoque Tradicional grupal siendo de 6.07, por lo que este enfoque queda ubicado en el tercer sitio respecto a los otros dos.

\section{Discusión y Conclusiones}

Dadas las características de esta investigación, la cual pretende describir parcialmente a partir de una muestra, la situación actual de la Educación Básica en México, en cuanto a la variable "Solución de Problemas", desde diferentes enfoques pedagógicos y basándonos en los resultados obtenidos se concluye lo siguiente:

- El enfoque pedagógico óptimo para la estimulación y la práctica de las "habilidades de pensamiento complejo" (Solución de Problemas), fue el enfoque "Freinet", en menor medida la escuela tipo Montessori y en una situación desventajosa, las escuelas con pedagogía Tradicional.

- Resulta indispensable incluir contenidos donde se ejercite, enseñe y desarrolle la S.P., como un aspecto fundamental en la Educación Básica, a la par de que se realicen investigaciones educativas encaminadas a identificar qué elementos son los indispensables para que se generen en el educando las "habilidades de pensamiento complejo" de manera adecuada.

- Las habilidades de pensamiento complejo, requieren por un lado, del tratamiento pedagógico adecuado (método), de la estimulación propicia (filosofía educativa), además, de una intervención adecuada y permisible (mentalidad y actitud docente) para que de este modo, las manifestaciones propias de cada individuo surjan sin ser ignoradas, reprimidas o castigadas.

- La escuela primaria que se rige bajo la pedagogía tradicional, en función de los resultados obtenidos en el presente trabajo, enseña en menor medida como resolver problemas en general, ( $\sin$ considerar los específicos en la asignatura de matemáticas). 
Al respecto ya se ha señalado: “...la solución de problemas debería constituir un contenido necesario de las diversas áreas del currículo obligatorio" (Pozo, et al.1994, p. $15)$.

- La educación primaria requiere con urgencia de una adecuada redefinición, (reconceptualización curricular), que abarque más allá de la interacción maestroalumno: es todo un estilo de enseñar y aprender, en un conjunto de estimulaciones y motivaciones, además de una manera diferente de percibirse y respetarse como educador, y de ver y respetar al educando, potenciando así todas sus capacidades.

- Podemos inferir a partir de los resultados de esta investigación, que el Sistema Educativo Nacional Mexicano presenta limitaciones en lo que se refiere a los recursos tanto humanos como materiales, dado que la motivación y la responsabilidad por parte del profesorado es un punto de análisis importante. Dentro del contexto de la problemática educativa, basándonos en Schmelkes (1994), y en sus aportaciones al respecto, concluye que el profesorado no se encuentra capacitado o bien está desinformado sobre su papel en la sociedad, por otro lado los recursos con los que cuentan también resultan ser insuficientes.

- Cualquier sociedad necesita escuelas donde se enseñe a pensar y a crear y no a repetir, o bien aceptar lo ya establecido. Los seres humanos somos "pensantes”, ¿por qué no llevarlo a la práctica educativa en nuestro país?

- Se requiere un nuevo modelo de enseñanza, que contemple todas las potencialidades del ser humano, pero lo más importante, que proporcione los recursos materiales y humanos necesarios para preparar, formar y capacitar óptimamente a todos los responsables de este proceso educativo, para que los cambios se observen y se vivan en la práctica, en las aulas y no sólo en el "papel” y que como una resultante, entonces sí, se permita la manifestación integral del educando, artículo $3^{\circ}$. (Constitución Política de los Estados Unidos Mexicanos 2000)

- Dentro de las principales características distintivas de la metodología de la escuela de tipo Freinet se encuentra: el diario escolar, correo interescolar, libro de la vida (imprenta en el salón de clases), los ficheros, conferencias (dar la palabra al niño), los huertos, los talleres (desarrollo de destrezas), asambleas, (posturas críticas) etc.

- Otra característica importante de la escuela Freinet consiste en fomentar la cooperación en el trabajo grupal. La interacción en grupos cooperativos como ya han señalado varios autores (Coll, 1997; Díaz Barriga y Hernández, 2001; Hernández, 
1998), tiene sus fundamentos en los postulados teóricos del paradigma sociocultural de Vygotsky.

- Sin embargo, una limitación de este estudio es la ausencia de control de algunas variables como la motivación, inteligencia, personalidad, rendimiento académico o profesor, que podrían explicar las diferencias observadas en S.P. entre los diferentes enfoques pedagógicos.

- Resaltamos que resultados muy parecidos a éstos se obtuvieron también en otro estudio en cuanto a la variable "creatividad" con la misma población (Esquivias y Muria, 2001), resaltando que la creatividad también es una habilidad del pensamiento complejo.

- Para finalizar consideramos importante mencionar que la escuela Freinet comparte muchos de los principios constructivistas de la teoría psicogenética de Piaget, así como de la teoría del aprendizaje significativo de Ausubel. Coll (1988; p.132), señala que uno de estos principios es el de la autoestructuración del conocimiento, "es decir, ven al alumno como el verdadero agente y el responsable último de su propio proceso de aprendizaje, 'como el artesano de su propia construcción' ".

- Consideramos que un análisis más profundo sobre la práctica educativa de la escuela Freinet puede contribuir enormemente a que los principios constructivistas se vean reflejados en acciones concretas en el aula y no se limiten a permanecer bellamente expresados sólo en los planes y programas del currículo vigente en nuestro país. 


\section{REFERENCIAS}

Acuña, E.C. y Batllori, G.A. (1988). Metacognición y Estrategias de Aprendizaje. El proceso de solución de problemas. Serie sobre la Universidad. Número 9. Centro de Investigación y Servicios Educativos: UNAM.

Ausubel, P. D., Novak, D. J. y Hanesian, H. (1998). Psicología Educativa. Un punto de vista cognoscitivo. México: Trillas.

Campbell, D. T. y Stanley, J. C. (1970). Diseños experimentales y cuasiexperimentales en la investigación Social. Buenos Aires: Amorrortu Editores.

Coll, C. (1988). Significado y sentido en el aprendizaje escolar. Reflexiones en torno al concepto de aprendizaje significativo. Infancia y Aprendizaje, 41, 131-142.

Coll, C. (1997). Constructivismo y educación escolar: ni hablamos siempre de lo mismo ni lo hacemos siempre de la misma perspectiva epistemológica. En M. J. Rodrigo y J. Arnay (Comp.) La construcción del conocimiento escolar. Barcelona: Paidós.

Constitución Política de los Estados Unidos Mexicanos. (2000). Leyes y Códigos de México. 131 . Edición. México: Editorial Porrúa.

Cook, T.D. y Campbell, D.F. (1979). Quasi-Experimentation Design \& Análisis Issues for Field Setting. Boston, U. S. A.: Houghton Mifflin Company.

De Vega, M. (1986). Introducción a la psicología cognitiva. México: Alianza.

Díaz Barriga, A. F. y Hernández, R. G. (2001). Estrategias Docentes para un aprendizaje significativo. Una interpretación constructivista. México: Editorial McGrawHill.

Esquivias, S. M. T. (1997). Estudio evaluativo de tres aproximaciones pedagógicas: Ecléctica, Montessori y Freinet, sobre la ejecución de solución de problemas y creatividad, con niños de escuela primaria. Tesis de Licenciatura. México. UNAM. Facultad de Psicología.

Esquivias, S. M. T. y Muria, V. I. (2001). Una evaluación de la creatividad en la Educación Primaria. Revista Digital Universitaria. UNAM. 14 de marzo de 2002. http://www.revista.unam.mx/vol.1/num.3/art1/.

Freinet, C. (1985). Técnicas Freinet de la Escuela Moderna. México: Siglo Veintiuno.

Hernández, R. G. (1998). Paradigmas en psicología de la educación. México: Paidós.

Hernández, S. R., Fernández C. C. y Baptista, L. P. (1991). Metodología de la Investigación. México: Mc Graw Hill.

Klausmeier, H. J. y Goodwin, W. (1993). Habilidades Humanas y Aprendizaje. México: Editorial Harla, S. A. 
Monés i Pujol-Busquets, J. (2000). María Montessori. En: Pedagogías del Siglo XX. Barcelona: Cuadernos de Pedagogía. Especial 25 años. Editorial CISSPRAXIS, S.A.

Vallet, M. (2000). María Montessori. En: Pedagogías del Siglo XX. Barcelona: Cuadernos de Pedagogía. Especial 25 años. Editorial CISSPRAXIS, S.A.

Vilaplana, E. (2000). Célestin Freinet. En: Pedagogías del Siglo XX. Barcelona: Cuadernos de Pedagogía. Especial 25 años. Editorial CISSPRAXIS, S.A.

Pozo, J. I. (1999). Teorías cognitivas del aprendizaje. Sexta Edición. Madrid: Editorial Morata.

Pozo, M. J. I., Pérez, E. M. del P., Domínguez, C. J., Gómez, C. M. A. y Postigo, A. Y. (1994). La solución de problemas. Madrid: Aula XXI Santillana.

Salkind, N. J. (1998). Métodos de Investigación. Tercera Edición. México: Prentice Hall.

Sánchez, M. A. (1996). Desarrollo de habilidades del pensamiento: razonamiento verbal y solución de problemas, guía del instructor. México: Trillas.

Schmelkes, S. (1994). La desigualdad en la calidad de la educación primaria. Resultados de un estudio realizado en Puebla. En: ¿Hacia dónde va la educación pública? Memorias del seminario de análisis sobre política educativa nacional. México: Fundación SENTE.

SEP. Programa para la Modernización Educativa. (1989-1994). México. Secretaría de Educación Pública. 


\section{Esta página está en blanco intencionadamente.}

